\title{
Preparation of monodisperse hybrid gel particles with various morphologies via flow rate and temperature control
}

Received 00th January 20xx, Accepted 00th January 20xx

\author{
Toshimitsu Kanai, ${ }^{* a}{ }^{\text {Hiroki Nakai, }}{ }^{a}$ Ayaka Yamada, ${ }^{a}$ Masafumi Fukuyama ${ }^{a}$ and David A. Weitz ${ }^{b}$
}

DOI: $10.1039 / \times 0 \times x 00000 x$

We report a facile method for preparing monodisperse hybrid smart gel particles with various morphologies by using microfluidic techniques and the swelling-shrinking phenomenon of thermosensitive poly( $\mathrm{N}$-isopropylacrylamide) (PNIPAM) gel particles. We demonstrate that PNIPAM-polyacrylamide snowman-like, raspberry-like, and dumbbell-like hybrid gel particles can be prepared by controlling the flow rate and temperature.

Hybrid particles, consisting of spheres with different size and chemical composition, are of increasing importance to fundamental research in colloidal and interface science, as well as to various potential applications in material science and engineering. They not only exhibit combined characteristics of their corresponding individual components, but also exhibit attractive features because of their morphologies: for example, snowman-like and dumbbell-like particles have anisotropic properties that can be applied in light scattering and fluid properties $^{1,2}$ and for engineering biomaterials ${ }^{3}$ and photonic crystals. ${ }^{4}$ They can also be used as colloid surfactants that can achieve more complex hierarchical structures and more effective stabilization compared with traditional surfactant molecules. ${ }^{5,6}$ Core-shell particles are highly functional materials with modified properties. Coating or encapsulating the core particle with the shell material enhances the stability and dispersibility and controls release of the core. ${ }^{7,8}$ Raspberry-like particles, consisting of smaller particles over a larger core particle, are promising materials as building blocks in the fabrication of superhydrophobic or superhydrophilic surfaces because of their high surface roughness and large surface area. ${ }^{9,10}$ Such hybrid particles, consisting of organic and/or inorganic materials, have been prepared with various methods including seeded emulsion polymerization, ${ }^{11-14}$ dynamic swelling method, ${ }^{15,16}$ phase separation, ${ }^{17-19}$ self-

\footnotetext{
${ }^{a}$ Dept. of Materials Science and Engineering, Yokohama National University, 79-5 Tokiwadai, Hodogaya, Yokohama, Kanagawa 240-8501, Japan. E-mail: tkanai@ynu.ac.jp

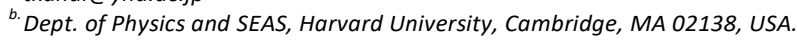
Electronic Supplementary Information (ESI) available. See DOI: $10.1039 / x 0 \times x 00000 x$
}

assembly, $^{20-22}$ and microfluidic processes. ${ }^{23,24}$ Recently, smart hydrogels have been receiving increasing attention because of their unique tunable properties. ${ }^{25-27}$ Hydrogels are threedimensional networks of polymer chains inflated with water, and their volume can be altered on demand by external stimuli. For example, poly( $N$-isopropylacrylamide) (PNIPAM) gel is one of the most important smart hydrogels that responds to the change in temperature close to human body. ${ }^{28,29}$ When heated over $32{ }^{\circ} \mathrm{C}$, the hydrophilic PNIPAM becomes hydrophobic, leading to shrinkage and release of its liquid contents. These properties are expected to be used as actuators for biological applications and controlled drug delivery. In most cases, hybrid particles with different morphologies are prepared by different methods. Recent studies have controlled particle morphology by adjusting the concentrations of the starting materials, e.g. monomers and crosslinker. ${ }^{11,13,30-32}$ This enables the preparation of hybrid particles with different morphologies in the same system. However, changing concentrations might restrict the properties of hybrid particles and is troublesome.

In this communication, we report a facile method for preparing monodisperse hybrid smart gel particles with various morphologies by using microfluidic techniques and swelling-shrinking phenomenon of PNIPAM gel particles. We demonstrate that snowman-like, raspberry-like, and dumbbelllike hybrid gel particles can be prepared using the same starting materials in the same system by varying only external factors, i.e. flow rate and temperature. Microfluidic devices consisting of networks of micron scale channels enable precise dimension control of the droplets generated. ${ }^{33-35}$ We show that monodisperse droplets of acrylamide (AAM) aqueous solution containing one or two PNIPAM gel particles can be generated through the microfluidic device. The core-shell structures can be transformed into snowman-like, raspberrylike, and dumbbell-like shapes thorough the swelling-shrinking phenomenon of PNIPAM gel particles by altering the temperature. In addition, we show that the volume of the part of PNIPAM in the PNIPAM-polyacrylamide (PAAM) hybrid gel particles can be altered by heating. 


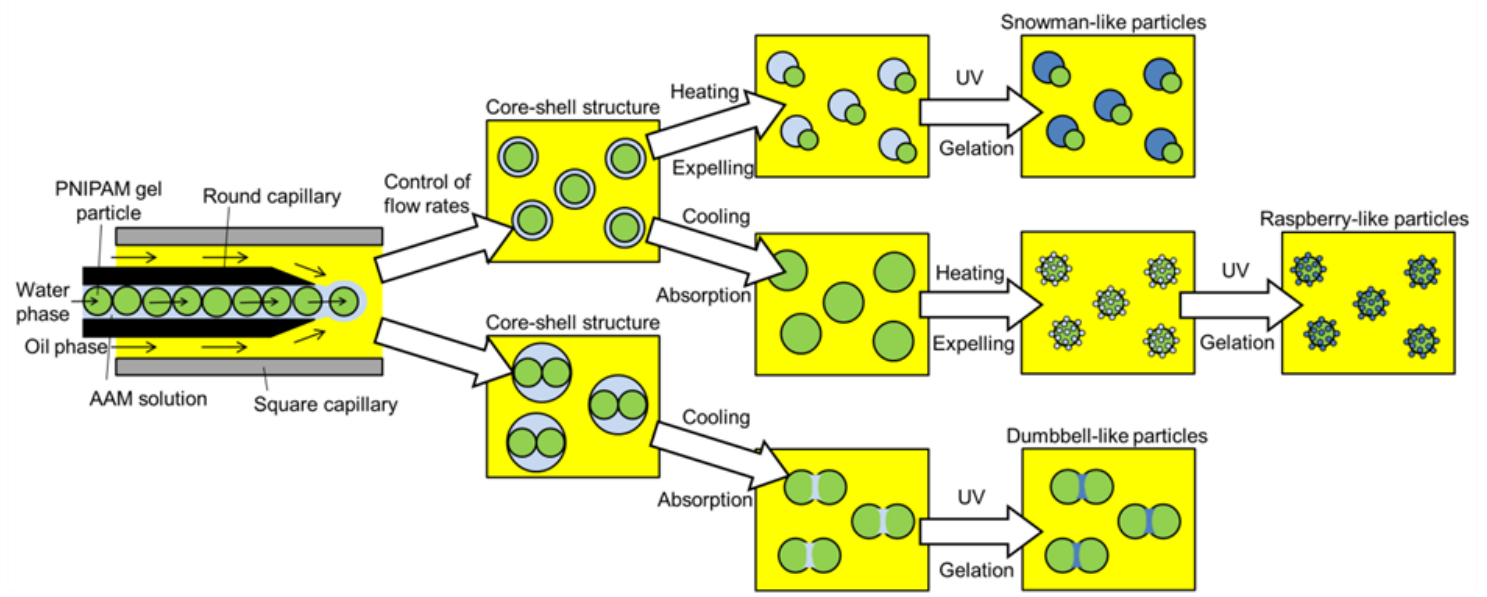

Fig. 1 Schematic representation of the process for the preparation of monodisperse PNIPAM-PAAM hybrid gel particles with various morphologies.

Fig. 1 shows the overall process for the preparation of monodisperse PNIPAM-PAAM hybrid gel particles with various morphologies (Information is listed with detail in the ESI+). As the first step, monodisperse PNIPAM gel particles are prepared by a combination of microfluidic techniques and photopolymerization. $^{36,37}$ The monodisperse PNIPAM gel particles obtained are dispersed in an AAM aqueous solution that is injected into the inner glass capillary with the inner diameter slightly larger than the diameter of the gel particles in the co-flow type microfluidic device, so that the PNIPAM gel particles are arranged in a row. The silicone oil containing a surfactant and a photoinitiator is injected into the outer square capillary in the device. By precisely controlling flow rates of the inner water and outer oil phases, monodisperse AAM aqueous droplets containing one or two PNIPAM gel particles are generated. When aqueous droplets containing one PNIPAM gel particle are heated, the gel particle shrinks and emerges from the droplets, resulting in a snowman-like shape. On the other hand, once AAM solution around the gel particle is absorbed fully into the gel particle by cooling and then expelled by heating, a raspberry-like shape is formed. When droplets containing two PNIPAM gel particles are cooled, the gel particles absorb the AMM solution to form a dumbbell-like shape. After these temperature controls, AAM solution is polymerized by UV light irradiation to prepare PNIPAM-PAAM hybrid gel particles with snowman-like, raspberry-like, and dumbbell-like morphologies.

We synthesized monodisperse PNIPAM gel particles by using a glass capillary microfluidic device with a flow-focusing geometry $^{38}$ (Fig. 2a). An aqueous solution containing $N$ isopropylacrylamide (NIPAM, $1 \mathrm{M}$ ) and $N, N^{\prime}$-methylene-bisacrylamide (BIS, $5 \mathrm{mM}$ ) was pumped from the one end of the outer square capillary, while silicone oil (50 cSt) containing a surfactant (2 wt\%, RSN-0749) and a photoinitiator (0.2 wt\%, DAROCUR 1173) was pumped from the opposite end into the orifice of the inner tapered collection capillary. The outer oil phase hydrodynamically accumulated the inner water phase, which broke into monodisperse aqueous droplets upon entering the collection capillary. After collection, the droplets were polymerized by UV light irradiation. The PNIPAM gel particles obtained were highly monodisperse, as shown in Fig. 2b. At least 100 PNIPAM gel particles (particle diameter: 147 $\mu \mathrm{m}$, coefficient of variation (CV): $3.4 \%$ ) together with an aqueous solution containing $\operatorname{AAM}(4 \mathrm{M})$ and BIS (100 mM) were filled into a round glass capillary with an inner diameter of $200 \mu \mathrm{m}$ in the co-flow type microfluidic device. Since the PNIPAM gel particles were moderately smaller than the inner diameter of the capillary, they could be arranged in a row (Fig. 2c-f). The water phase was pumped from the inner to outer capillary in the device, whereas the silicone oil
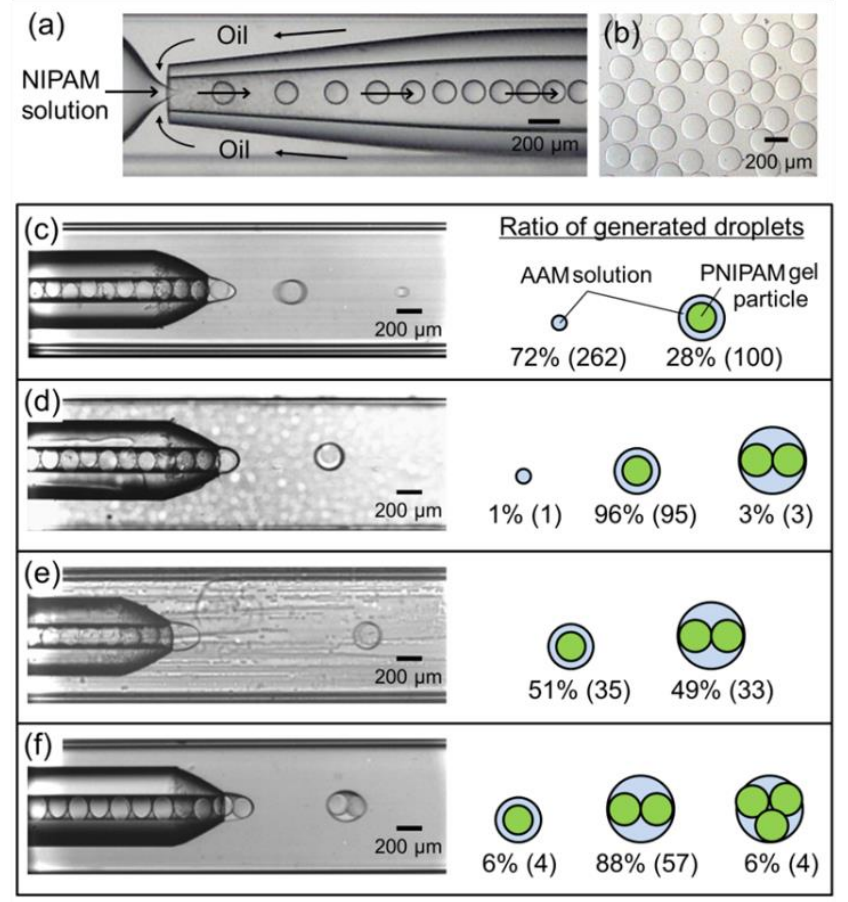

Fig. 2 (a) NIPAM solution monodisperse droplet formation in a flow-focusing microfluidic device. Flow rates of the inner water and outer oil phases are 3.0 and $0.4 \mathrm{~mL} \mathrm{~h}^{-1}$ respectively; (b) Optical micrograph of the prepared
monodisperse PNIPAM gel particles in isopropyl alcohol; (c)-(f) Generation of droplets containing PNIPAM gel particles in a co-flow microfluidic device at the following flow rates of the outer oil phase when the flow rate of the inner Whe number of each droplet generated is shown in parentheses. 

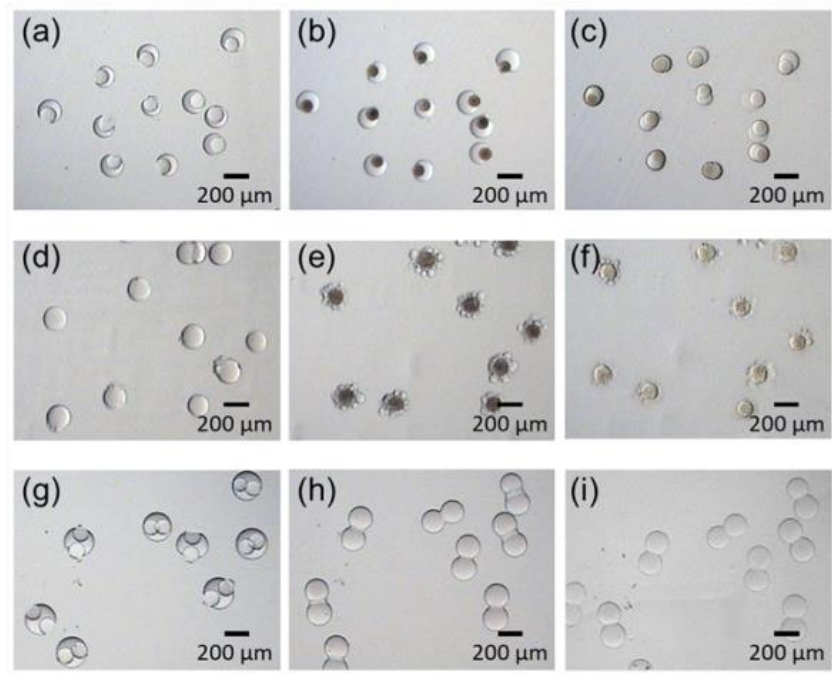

Fig. 3 (a)-(b): Photograph of the droplets containing one PNIPAM gel particle (a) before and (b) after heating to $45^{\circ} \mathrm{C}$; (c): Photograph of the PNIPAM-PAAM snowman-like hybrid gel particles prepared by UV light irradiation; (d)-(e): $8^{\circ} \mathrm{C}$ and (e) subsequently heated to $45^{\circ} \mathrm{C}$; ( $\mathrm{f}$ ): Photograph of the PNIPAM$8^{\circ} \mathrm{C}$ and (e) subsequently heated to $45^{\circ} \mathrm{C}$; ( $\mathrm{f}$ ): Photograph of the PNIPAM(h): Photograph of the droplets containing two PNIPAM gel particles (g) before and $(\mathrm{h})$ after cooling to $8{ }^{\circ} \mathrm{C}$; (i): Photograph of the PNIPAM-PAAM dumbbelllike hybrid gel particles prepared by UV light irradiation.

containing the surfactant and photoinitiator was pumped to the outer capillary in the same direction. The outer oil phase fragmented the inner water phase into droplets at the outlet of the inner capillary. We found that the number of the gel particles in the droplets could be controlled by changing the flow rates. For example, droplets with and without the PNIPAM gel particle were generated when the flow rates of the inner water and outer oil phases were 8 and $290 \mathrm{~mL} \mathrm{~h}^{-1}$, respectively (Fig. 2c). When the flow rate of the inner phase was maintained constant at $8 \mathrm{~mL} \mathrm{~h}^{-1}$, the ratio of the gelexcluded droplet decreased as the flow rate of the outer phase was decreased. At an outer oil phase flow rate of 270 $\mathrm{mL} \mathrm{h}^{-1}$, almost all generated droplets (96\%) contained one PNIPAM gel particle (Fig. 2d). Upon decreasing the flow rate of the outer phase further, the droplet containing two gel particles increased (Fig. 2e). Eventually, almost all generated droplets (88\%) contained two PNIPAM gel particles at an outer phase flow rate of $160 \mathrm{~mL} \mathrm{~h}^{-1}$ (Fig. 2f).

When the droplets containing one PNIPAM gel particle were heated to $45{ }^{\circ} \mathrm{C}$, the gel particles shrank and expelled the liquid content (Fig. $3 a$ and b). Since the PNIPAM gel turned from hydrophilic to hydrophobic, particles emerged from the AAM aqueous droplets, resulting in the snowmanlike shape. When the AAM droplets were polymerized by UV light irradiation, PNIPAM-PAAM snowman-like hybrid gel particles were prepared (Fig. 3c). On the other hand, when the droplets were cooled to $8{ }^{\circ} \mathrm{C}$, the PNIPAM gel particle fully absorbed the surrounding AAM solution (Fig. $3 \mathrm{~d}$ ). Upon heating them to $45{ }^{\circ} \mathrm{C}$, a lot of small AAM droplets were expelled from all over the interface of the gel particles during their shrinkage (Fig. 3e). The small droplets coalesced at a low surfactant concentration. When the concentration was sufficiently high, i.e. 2 wt\%, the raspberry-like shape was maintained. When the droplets were photopolymerized,

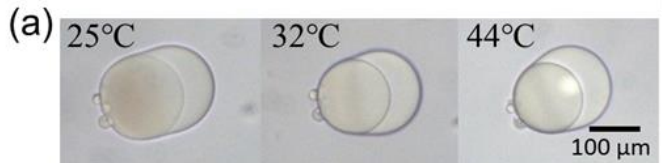

(b)
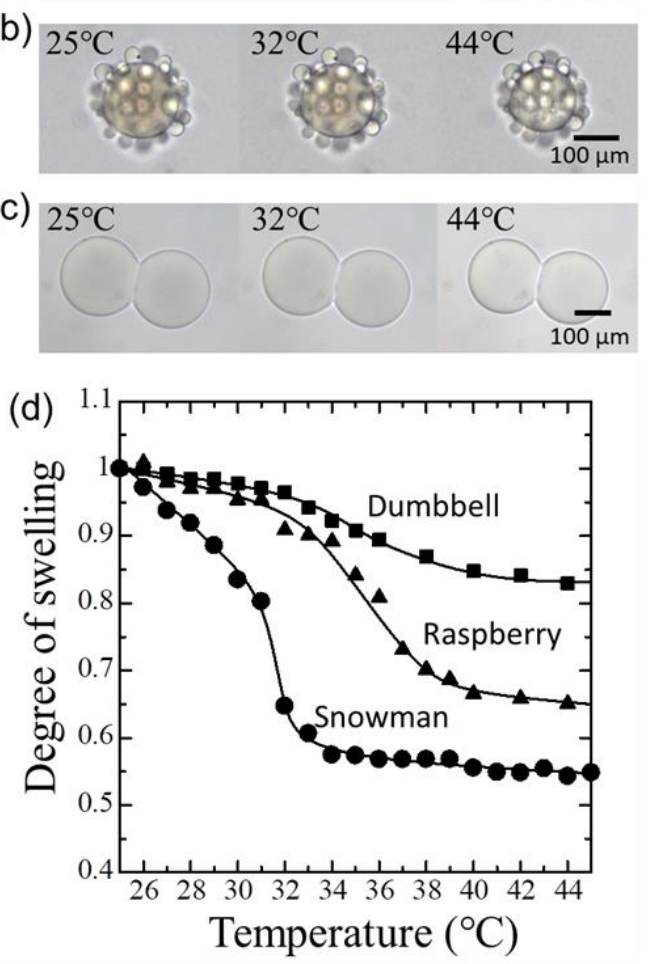

Fig. 4 Photographs of (a) snowman-like, (b) raspberry-like and (c) dumbbelllike hybrid gel particles at the representative temperatures. Photographs are Degree of swelling of PNIPAM gel particles in hybrid gel particles as a function of temperature.

PNIPAM-PAAM raspberry-like hybrid gel particles were prepared (Fig. 3f). In addition, when droplets containing two PNIPAM gel particles were cooled to $8{ }^{\circ} \mathrm{C}$, gel particles absorbed the surrounding AAM solution. However, a small amount of solution remained between the particles, resulting in the dumbbell-like shape (Fig. $3 g$ and h). Thus, the dumbbell-like hybrid gel particles were prepared by the photopolymerization of the AAM solution (Fig. 3i). These hybrid particles were stable and did not aggregate after being washed and placed in water.

Since PNIPAM gel is characterized by temperature responsivity, the volume of the PNIPAM part in the hybrid gel particles can be altered by heating. As the temperature of the snowman-like hybrid gel particles was increased, PNIPAM gel particles began to shrink at around $32{ }^{\circ} \mathrm{C}$ while PAAM gel particles did not (Fig. 4a). Upon further increasing temperature, the volume and the swelling ratio of the PNIPAM gel particle remained constant, as expected for PNIPAM gel. The similar shrinkage behaviour was observed in the raspberry-like and dumbbell-like hybrid gel particles (Fig. $4 \mathrm{~b}$ and $\mathrm{c}$ ). The constituent particles did not separate after temperature changes. Moreover, the corresponding volume changes showed good repeatability; the variability of the volume was less than $5 \%$. The volume of the PNIPAM gel 
particles in the snowman-like hybrid gel particles ultimately decreased to about $55 \%$ of the initial one (Fig. $4 d$ ). On the other hand, the degree of the shrinkage in the raspberry-like and dumbbell-like hybrid gel particles was smaller than that in the snowman-like hybrid gel particles. This is because more PAAM chains tangled with the network of the PNIPAM gel, and it interfered with the shrinkage of the PNIPAM.

\section{Conclusions}

In summary, we have demonstrated a facile method for the preparation of monodisperse hybrid smart gel particles with various morphologies by using microfluidic techniques and swelling-shrinking phenomenon of PNIPAM gel particles. We found that monodisperse droplets containing one or two PNIPAM gel particles could be generated in the microfluidic device, and they could be transformed into the snowman-like, raspberry-like, and dumbbell-like shapes by temperature control. In addition, we showed that the volume of the PNIPAM part in the hybrid gel particles could be altered by heating. Although the size of hybrid gel particles prepared in this study was several hundred micrometres, the size could be reduced to several hundred nanometres by reducing the dimension of the flow channels in the device. While we demonstrated the preparation of PNIPAM-PAAM hybrid gel particles, this principle should also be applicable to prepare hybrid gel particles consisting of other smart hydrogels. Hybrid gel particles with various morphologies have a great potential for tunable and multifunctional materials in material science and bioengineering.

\section{Conflicts of interest}

There are no conflicts to declare.

\section{Notes and references}

1 M. I. Mischenko, J. W. Hovenier and L. D. Travis, Light scattering by nonspherical particles: Theory, measurements, applications, Academic Press, San Diego, 2000.

2 R. G. Larson, The structure and rheology of complex fluids, Oxford University Press, New York, 1998.

3 H. C. Shum, A. R. Abate, D. Lee, A. R. Studart, B. G. Wang, C. H. Chen, J. Thiele, R. K. Shah, A. Krummel and D. A. Weitz, Macromol. Rapid Commun., 2010, 31, 108.

4 I. D. Hosein, M. Ghebrebrhan, J. D. Joannopoulos and C. M. Liddell, Langmuir, 2010, 26, 2151.

5 J. W. Kim, R. J. Larsen and D. A. Weitz, J. Am. Chem. Soc., 2006, 128, 14374

6 J. W. Kim, D. Lee, H. C. Shum and D. A. Weitz, Adv. Mater., 2008, 20, 3239.

7 R. G. Chaudhuri and S. Paria, Chem. Rev., 2012, 112, 2373.

8 F. Caruso, Adv. Mater., 2001, 13, 11.

9 H. J. Tsai and Y. L. Lee, Langmuir, 2007, 23, 12687.

10 X. Du, X. Liu, H. Chen and J. He, J. Phys. Chem. C, 2009, 113, 9063.

11 X. Pei, K. Zhai, X. Liang, Y. Deng, K. Xu, Y. Tan, X. Yao and P. Wang, J. Colloid Interf. Sci., 2018, 512, 600.

12 J. W. Kim and K. D. Suh, J. Ind. Eng. Chem., 2008, 14, 1.
13 F. W. Wang, H. R. Liu, L. L. Zhao and X. Y. Zhang, Colloid Polym. Sci., 2014, 292, 1171.

14 A. Perro, S. Reculusa, E. Bourgeat-Lami, E. Duguet and S. Ravaine, Colloids Surf., 2006, 284, 78.

15 M. Okubo, T. Yamashita, H. Minami and Y. Konishi, Colloid Polym. Sci., 1998, 276, 887.

16 W. K. Kegel, D. Breed, M. Elsesser and D. J. Pine, Langmuir, 2006, 22, 7135

17 Z. Luo, Y. Li and B. Liu, Chem. Commun., 2017, 53, 8649.

18 Q. Niu, M. W. Pan, J. F. Yuan, X. Liu, X. M. Wang and H. F. Yu, Macromol. Rapid Commun., 2013, 34, 1363.

19 X. Liu, M. W. Pan, J. F. Yuan, Q. Niu, X. M. Wang and K. C. Zhang, RSC Adv., 2014, 4, 4163.

20 K. Zhang, M. Jiang and D. Chen, Prog. Polym. Sci., 2012, 37, 445.

21 S. Sacanna, M. Korpics, K. Rodriguez, L. Colon-Melendez, S.H. Kim, D. J. Pine and G.-R. Yi, Nat. Commun., 2013, 4, 1688.

22 H. Y. Koo, D. K. Yi, S. J. Yoo and D. Y. Kim, Adv. Mater., 2004, 16, 274.

23 D. Dendukuri and P. S. Doyle, Adv. Mater., 2009, 21, 4071.

24 T. Kanai, D. Lee, H. C. Shum, R. K. Shah and D. A. Weitz, Adv. Mater., 2010, 22, 4998.

25 C. Yao, Z. Liu, C. Yang, W. Wang, X. J. Ju, R. Xie and L. Y. Chu, ACS Appl. Mater. Interfaces, 2016, 8, 21721.

26 T. Miyata, N. Asami and T. Uragami, Nature, 1999, 399, 766.

27 A. S. Hoffman, Adv. Drug Delivery Rev., 2012, 64, 18.

28 M. Zhang, C. C. Song, R. Ji, Z. Y. Qiao, C. Yang, F. Y. Qiu, D. H. Liang, F. S. Du and Z. C. Li, Polym. Chem., 2016, 7, 1494.

29 H. Sugiyama, T. Sawada, H. Yano and T. Kanai, J. Mater. Chem. C, 2013, 1, 6103.

30 B. Yu, H. Yuan, D. Wang, H. Cong, X. Xu and S. Yang, Colloid Polym. Sci., 2014, 292, 2361.

31 J. Yuan, L. Wang, L. Zhu, M. Pan, W. Wang, Y. Liu and G. Liu, Langmuir, 2015, 31, 4087.

32 Z. Zhang, H. Shao, X. Zhou, L. Zhao, H. Liu, X. Ji and H. Liu, Mater. Chem. Phys., 2017, 195, 105.

33 L. Y. Chu, A. S. Utada, R. K. Shah, J. W. Kim and D. A. Weitz, Angew. Chem., Int. Ed., 2007, 46, 8970.

34 T. Kanai and M. Tsuchiya, Chem. Eng. J., 2016, 290, 400.

35 J. J. Tan, C. M. Li, H. Li, H. Zhang, J. W. Gu, B. L. Zhang and Q. Y. Zhang, Polym. Chem., 2015, 6, 4366.

36 T. Kanai, D. Lee, H. C. Shum and D. A. Weitz, Small, 2010, 6 , 807.

37 T. Kanai, K. Ohtani, M. Fukuyama, T. Katakura and M. Hayakawa, Polymer J., 2011, 43, 987.

38 A. S. Utada, E. Lorenceau, D. R. Link, P. D. Kaplan, H. A. Stone and D. A. Weitz, Science, 2005, 308, 537. 